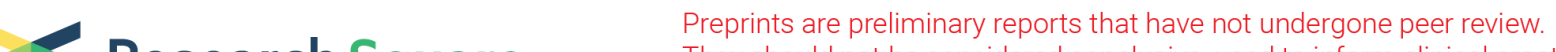 Research Square
They should not be onsidideded conllusive, used to inform clinical practice,
or referenced by the media salidated information.
}

\section{An Unusual Cause of Failure In Zenith Alpha Abdominal Endograft}

\section{Raffaella N Berchiolli}

Azienda Ospedaliero Universitaria Pisana

Michele Marconi ( $\Delta$ michemarconi@gmail.com )

AOUP: Azienda Ospedaliero Universitaria Pisana https://orcid.org/0000-0002-3291-5059

Irene Bargellini

AOU Pisana: Azienda Ospedaliero Universitaria Pisana

\section{Giulia Bertagna}

Azienda Ospedaliero Universitaria Pisana

\section{Daniele Adami}

Azienda Ospedaliero Universitaria Pisana

Davide M Mocellin

Azienda Ospedaliero Universitaria Pisana

Roberto Cioni

Azienda Ospedaliero Universitaria Pisana

Mauro Ferrari

Azienda Ospedaliero Universitaria Pisana

\section{Case report}

Keywords: Abdominal aortic aneurysm, Endoleak, Device design, Endovascular aneurysm repair, Endovascular treatment/therapy

Posted Date: May 26th, 2021

DOI: https://doi.org/10.21203/rs.3.rs-540343/v1

License: (9) This work is licensed under a Creative Commons Attribution 4.0 International License.

Read Full License

Version of Record: A version of this preprint was published at European Journal of Medical Research on March 2nd, 2022. See the published version at https://doi.org/10.1186/s40001-022-00656-5. 


\section{Abstract}

\section{Background}

The disruption of the stent component of the graft is an unusual complication of the endovascular abdominal aortic aneurysm repair (EVAR).

Case presentation

A 71-year-old man underwent EVAR followed by initial significant sac shrinkage. At 24 months, ultrasound and computed tomography showed sac growth associated with suprarenal stent graft separation from main body, without evidence of endoleak. A thoracic endograft was placed with complete relining of the previous graft.

Conclusions.

The report demonstrates un uncommon cause of endograft failure with suprarenal stent separation from a Zenith Cook main body and highlights the need for continuous follow-up.

\section{Background}

Endovascular aneurysm repair (EVAR) has been widely recognized as an alternative to open surgery for the treatment of abdominal aortic aneurysm (AAA), because it presents lower early morbidity and mortality rates, even if secondary interventions and late complications are more common.

Here, we describe an unusual case of disconnection of the bare suprarenal stent from the main body of a Zenith Alpha endograft, which led to enlargement of the sac without evidence of endoleak.

The patient's consent was obtained for the publication of this report.

\section{Case Presentation}

A 71-year-old man with an enlarging infrarenal AAA ( $63 \mathrm{~mm}$ of maximum diameter) underwent EVAR using the Zenith Alpha Abdominal graft (Cook Medical Bloomington, IN, USA) under general anesthesia in May 2018.

The aneurysm characteristics were within the instructions for use (IFU) of the Zenith Cook device (ZC): infrarenal aortic neck length was $16 \mathrm{~mm}$ with a maximum angulation of $10^{\circ}$, neck diameter $23 \mathrm{~mm}$, no severe calcifications or thrombus. The diameters of the common iliac arteries were $16 \mathrm{~mm}$ on the right side and $14 \mathrm{~mm}$ on the left.

The main body ZIMB-26-84 was deployed via a left open femoral access with an homolateral iliac extension (Zenith Flex ZSLE-16-56 ZT) and an extension was added to the right iliac limb (ZSLE-20-56 $\mathrm{ZT}$ ). No complications occurred during the deployment or the retrieval of the main body device. There 
was no endoleak at the final angiography after Reliant ballooning (Medtronic Vascular, Santa Rosa, INC, USA).

The patient had an uneventful recovery and was discharged 2 days later.

The 4-weeks postoperative computed tomography (CT) scan showed complete exclusion of the aneurysm without complications (Fig. 1).

Follow-up duplex ultrasound (US) at 6,12 and 18 months demonstrated a progressive aneurysm sac shrinkage from $63 \mathrm{~mm}$ to $38 \mathrm{~mm}$ without signs of endoleak. However, at 24 months, duplex US revealed sac enlargement (54 $\mathrm{mm}$ ) without endoleak evidence.

CT was performed, showing the disconnection of the bare fixation suprarenal stent from the main body stent-graft that migrated distally and a severe kinking of the left iliac graft, without any evidence of endoleak (Fig. 2).

Given the aneurysm growth, an endovascular relining procedure was scheduled. Since the distance to the bifurcation was sufficient, a Zenith TX2 thoracic endograft (TBE-28-80-PF) was selected as a lining device. A Smart Control Stent $12 \times 60 \mathrm{~mm}$ was placed into the left leg of the bifurcated graft to correct the kinking.

The postoperative CT scan revealed that the right renal artery was partially covered by the prosthesis (Fig. 3). In order to prevent an occlusion, a bare metal stent (RX HERCULINK Elite Renal Stent System $5 \times 15$ mm; Abbott CardioVascular, Plymouth, MN, USA) was successfully deployed. After 6 months, the aneurysm sac was stable.

\section{Discussion}

EVAR is a minimally invasive modality for AAA treatment associated with a reduced perioperative mortality rate compared to open repair. For those reasons EVAR has become the preferred option in infrarenal AAA repair, but long-term follow-up suggests that the survival benefit from EVAR is lost, due to a higher rate of reinterventions [1].

The causes of EVAR failure are multifactorial, depending upon anatomy features, inaccurate preimplantation planning and deployment or device failure.

The ZC endograft has demonstrated a durable repair of AAA, with a low aneurysm-related mortality and an acceptable rate of reinterventions.

However, the junction between the suprarenal bare stent and the proximal part of the main body the graft may represent an area of weakness, and disconnections of the proximal uncovered stent have been reported with the first generation of this device prior to 2002 [2-3]. At that time, the stent graft was modified with a double-suture reinforcement to secure the uncovered stent. Since this modification was 
introduced, uncovered stent disconnection has become very rare, as even reported by Torres-Blanco et al. and Lindstrom et al. [4-5].

So far, no cases of disconnections of the newest generation of ZC devices, the Zenith Alpha, have been reported. Our case, however, shows that this issue has not definitively been resolved with this new generation of endograft.

In our case, despite an initial AAA sac shrinkage, the disconnection occurred only two years after EVAR, while previous reports have shown this complication to occur $3-8$ years after treatment [4-7].

Careful re-review of preoperative planning confirmed endograft sizing within the manufacturer's instructions for use and the anatomy gives no direct hints on why this endograft failed. The endograft implantation was retrospectively analyzed step-by-step without evidence of problems. The proximal site of sealing was ballooned within the first covered stent, and the balloon was inflated in accordance with its IFU.

This case has been reported to Cook Medical, in order to improve current products and future designs, and an investigation has been initiated.

In our case, no endoleak was detected at imaging, although the evident sac enlargement represented the sign of the endotension caused by the failed proximal seal.

A thoracic endograft was used in this case to span the distance from renal artery to the bifurcation of main body and obtain a complete relining. The length of the thoracic graft was $80 \mathrm{~mm}$, and it seemed to be a more appropriate device to ensure the maximum overlap and the greatest columnar strength.

\section{Conclusion}

Despite the improvements of the device, disconnection of the suprarenal stent in Zenith Alpha stentgrafts is a possible, yet rare complication, not having been previously reported. This complication underlines the need for a continued lifelong surveillance after EVAR, even with the use of newest generation devices.

\section{Declarations}

Declarations of interest: None

Meeting: This work has not been presented before.

Ethics approval and consent to participate: Not applicable

Consent for publication: Written informed consent for publication of their clinical details and clinical images was obtained from the patient. A copy of the consent form is available for review by the Editor of 
this journal.

Availability of data and materials: The datasets generated and analysed during the current study are available from the corresponding author on reasonable request.

Competing interests: The authors declare that they have no competing interests

Funding: No funding

Authors' contributions: All authors partecipated equally to the final form of the manuscript. All the authors read and approved the final manuscript

Acknowledgements: Not applicable

\section{References}

1. Sharma A, Sethi P, Gupta K. Endovascular Abdominal Aortic Aneurysm Repair Interv Cardiol Clin. 2020 Apr;9(2):153-168.

2. Ghanim K, Mwipatayi BP, Abbas M, Sieunarine K. Late stent-graft migration secondary to separation of the uncovered segment from the main body of a Zenith endoluminal graft. J Endovasc Ther. 2006; 13(3): 346-349.

3. Greenberg RK, Chuter TAM, Cambria RP, Sternbergh WC, Fearnot NE. Zenith abdominal aortic aneurysm endovascular graft. J Vasc Surg. 2008 Jul;48(1):1-9.

4. Torres-Blanco A, Molina-Nacher V, Sala-Almonacil V, Ortiz-Monzón E. A Rare Complication After Endovascular Aneurysm Repair: Disconnection of the Suprarenal Stent of a Zenith Endograft. J Endovasc Ther. 2016 Apr;23(2):307-10.

5. Lindström D, Wahlgren CM, Sonesson B, Resch T. Disintegration of the Top Stent on Zenith Abdominal Aortic Stent-Grafts. J Endovasc Ther. 2016 Apr;23(2):302-6.

6. Ueda T, Tajima H, Murata S, Iwata K, Saitou H, Miki I, Yasui D, Sugihara F, Onozawa S, Morota T, Kumita S. An Extremely Rare Complication: Abdominal Aortic Aneurysm Rupture Caused by Migration of a Zenith Main Body Years After Repair of the Suprarenal Stent Separation. J Endovasc Ther. 2019 Apr;26(2):269-272.

7. Oderich GS, Roeder B. Commentary: Proximal Uncovered Stent Disconnections With the Standard and Low-Profile Zenith AAA Stent-Grafts. J Endovasc Ther. 2016 Apr;23(2):311-3.

\section{Figures}




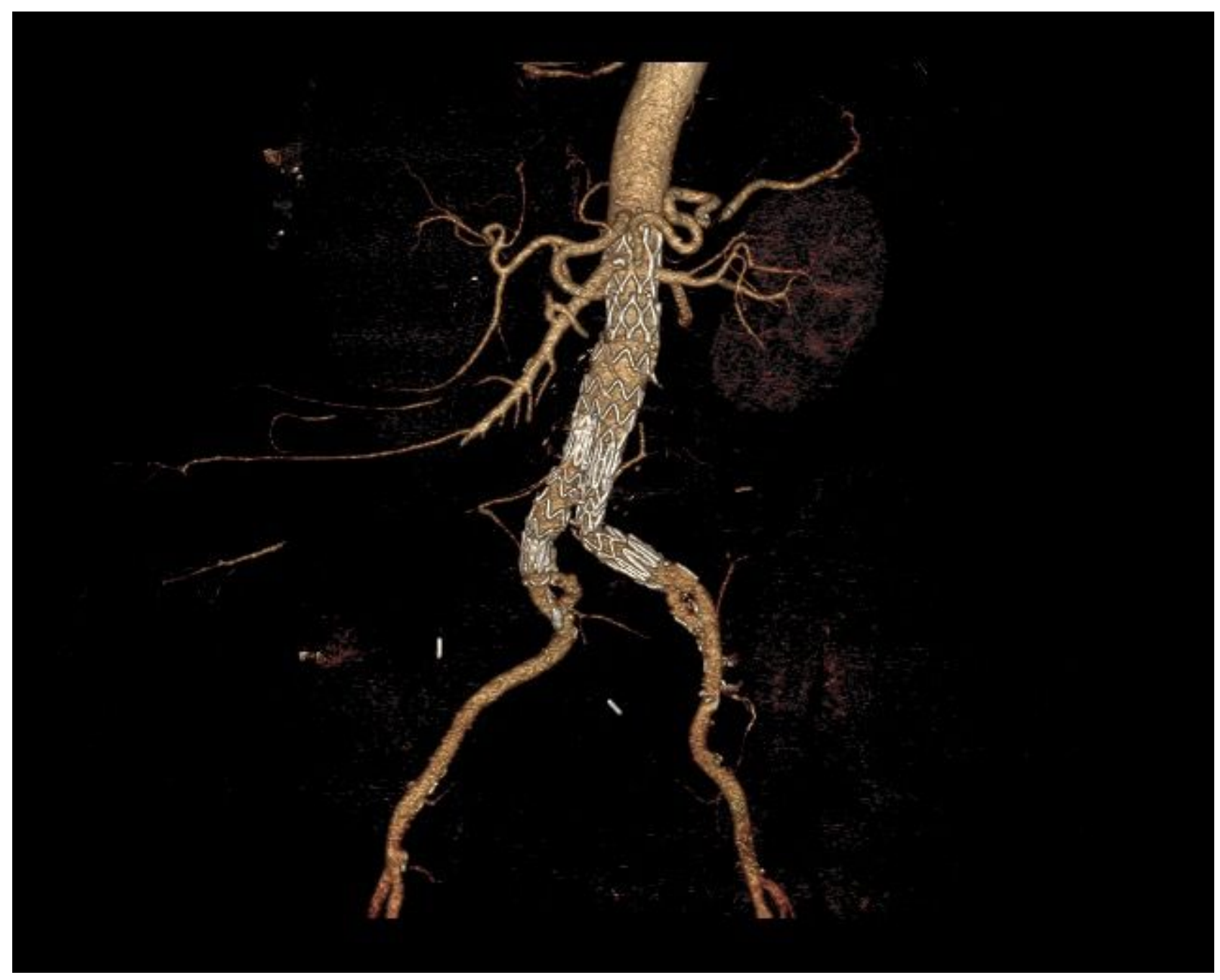

Figure 1

Postoperative CT scan after initial EVAR 


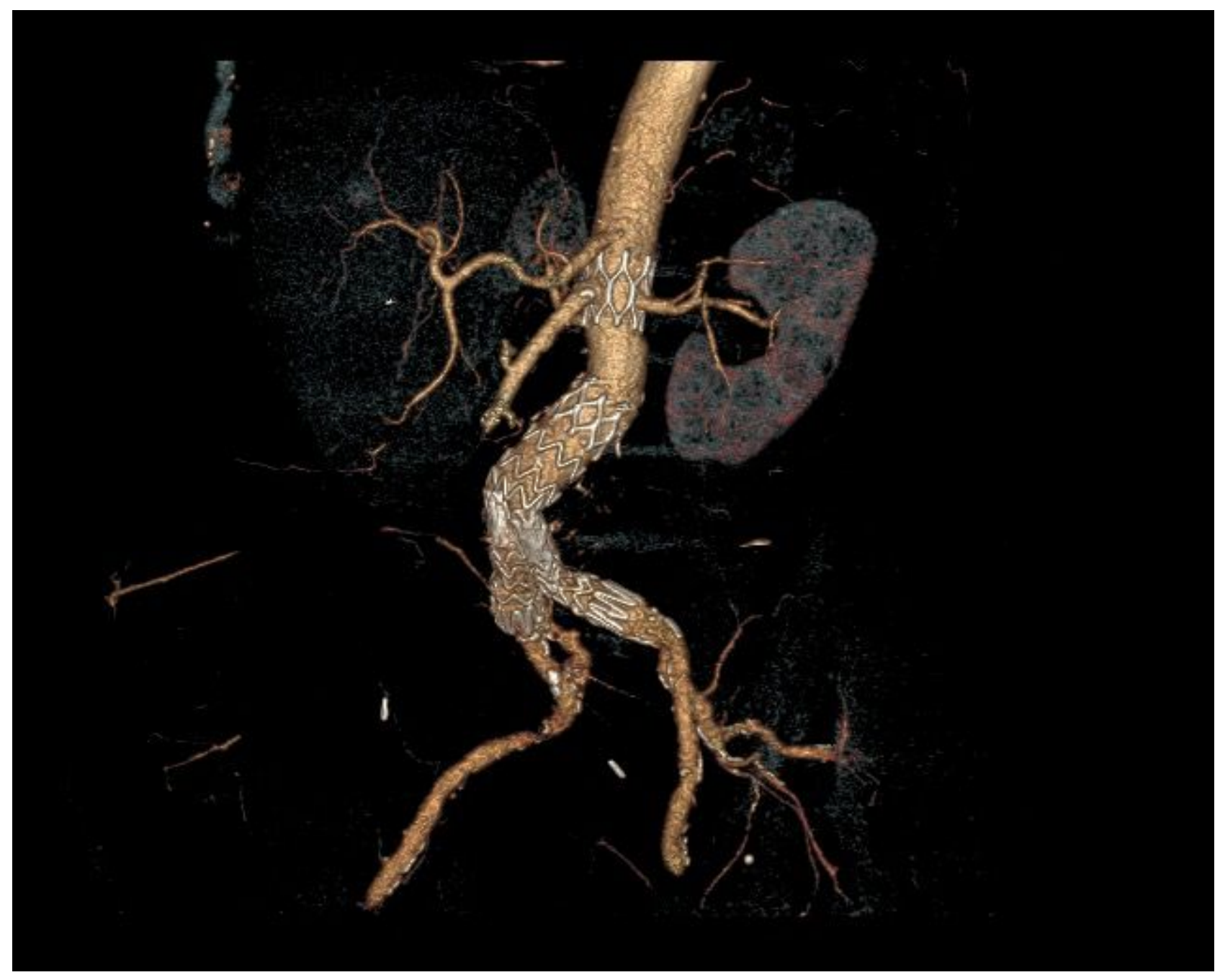

\section{Figure 2}

24-month CT scan showing disconnection of the bare suprarenal stent from main body stent-graft 


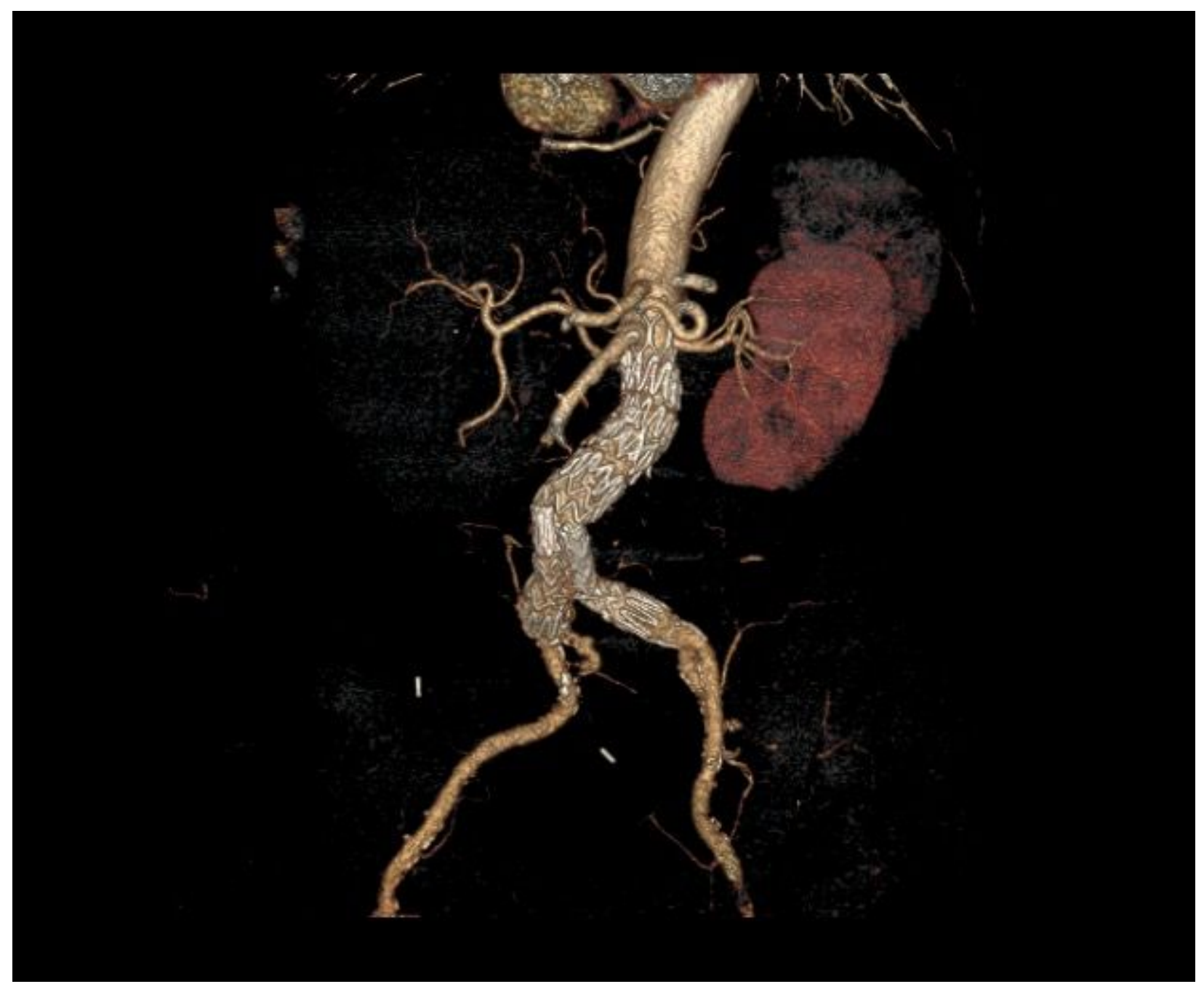

Figure 3

Postoperative CT scan after EVAR correction 